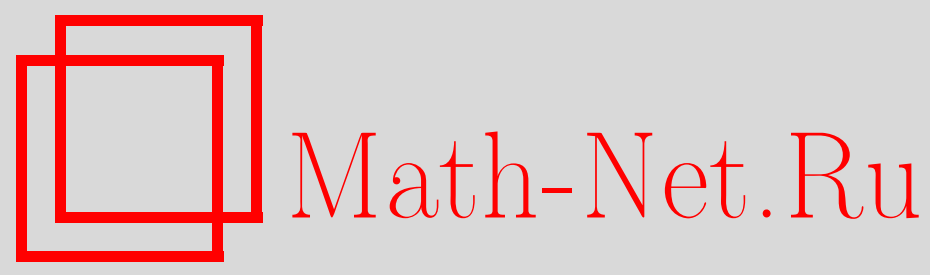

В. А. Юдин, Наименее уклоняющиеся от нуля многочлены, Матем. заметки, 2005, том 78, выпуск 2, 308-313

DOI: https://doi.org/10.4213/mzm2587

Использование Общероссийского математического портала Math-Net.Ru подразумевает, что вы прочитали и согласны с пользовательским соглашением http://www . mathnet.ru/rus/agreement

Параметры загрузки:

IP : 3.89.185.249

26 апреля 2023 г., 11:21:58

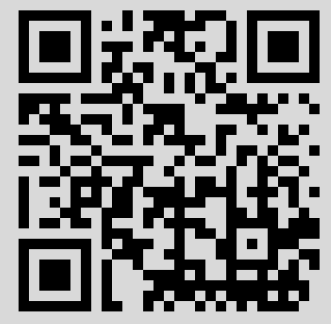


УДК 517.5

\section{НАИМЕНЕЕ УКЛОНЯЮЩИЕСЯ ОТ НУЛЯ МНОГОЧЛЕНЫ}

\section{В. А. Юдин}

На единичном круге $B$ определяется новое семейство многочленов, наименее уклоняющихся от нуля. Даются оценки снизу наилучших приближений в пространстве $L_{p}(B), p \geqslant 1$.

Библиография: 10 названий.

Через $B$ обозначим единичньй круг $B=\left\{(x, y) \in \mathbb{R}^{2}: x^{2}+y^{2} \leqslant 1\right\}$ с границей Г. Рассмотрим однородный многочлен порядка $n$

$$
f(x, y)=\sum_{k=0}^{n} a_{k} x^{k} y^{n-k}, \quad a_{k} \in \mathbb{R},
$$

и двумерный вариант задачи Чебьшева [1] для круга: найти наилучшее приближение $f(x, y)$ алгебраическими многочленами меньшей степени, т.е. многочленами из множества

$$
\mathscr{P}_{n-1}=\left\{P(x, y): P(x, y)=\sum_{k+l \leqslant n-1} C_{k, l} x^{k} y^{l}\right\} .
$$

Требуется вьчислить

$$
E_{n-1}(f)=\inf _{P \in \mathscr{P}_{n-1}}\|f(x, y)-P(x, y)\|=\inf _{P \in \mathscr{P}_{n-1}} \max _{(x, y) \in B}|f(x, y)-P(x, y)| .
$$

В некоторых частных случаях задача сводится к одномерной [1]; например, при $f(x, y)=$ $(a x+b y)^{n}$

$$
E_{n-1}(f)=2^{1-n}\left(a^{2}+b^{2}\right)^{n / 2}, \quad n \in \mathbb{N} .
$$

Существенное продвижение было сделано в [2], где уклонение (2) вычислено для некоторого класса многочленов (1). В частности,

$$
E_{n+m-1}\left(x^{n} y^{m}\right)=2^{1-n-m} \text {. }
$$

Оценки снизу получены методом экстремальных сигнатур [3], а приближающие многочлены строятся с помощью многочленов Чебьшева второго рода

$$
U_{k}(t)=\frac{\sin (k+1) \alpha}{\sin \alpha}, \quad t=\cos \alpha .
$$

Работа выполнена при финансовой поддержке Российского фонда фундаментальных исследований, грант № 05-01-00986. 
Для вычисления $(3)$ построение $P(x, y) \in \mathscr{P}_{n+m-1}$ происходит так, чтобы

$$
x^{n} y^{m}-P(x, y)=2^{-n-m}\left(U_{n}(x) U_{m}(y)+U_{n-2}(x) U_{m-2}(y)\right) .
$$

В настоящей статье мы выделим новый класс многочленов из (1), для которых уклонение (2) точно вычисляется. Рассматриваемая задачапредставляет интерес и в других пространствах. Получим нижние оценки $(2)$ в $L_{p}(B), p \geqslant 1$, с нормой

$$
\|f(x, y)\|_{p}=\left\{\frac{1}{\pi} \iint_{B}\left|f(x, y)^{p} d x d y\right|\right\}^{1 / p} .
$$

На их основе найдем точные значения уклонений в $L_{p}(B)$ произвольного однородного гармонического многочлена от подпространства $\mathscr{P}_{n-1}$. В отличие от методов [2], [4] будем использовать простые, хорошо известные (они встречаются еше у Бернштейна [5], см. также [6]) соображения относительно тригонометрических полиномов. Пусть

$$
t(\varphi)=\sum_{|\nu|<n} t_{\nu} e^{i \nu \varphi}, \quad\|t\|_{p}=\left\{\frac{1}{2 \pi} \int_{0}^{2 \pi}|t(\varphi)|^{p} d \varphi\right\}^{1 / p}, \quad p \geqslant 1
$$

тогда для любого $t(\varphi)$

$$
\left\|a_{n} e^{i n \varphi}+a_{-n} e^{-i n \varphi}-t(\varphi)\right\|_{p} \geqslant\left\|a_{n} e^{i n \varphi}+a_{-n} e^{-i n \varphi}\right\|_{p}
$$

Наилучшее приближение старшей гармоники меньшими осушествляется тривиально, т.е. $t(\varphi) \equiv 0$.

Перейдем в (1) к полярной системе координат, положим $x=\rho \cos \varphi, y=\rho \sin \varphi$. Из формул Эйлера получим

$$
\begin{aligned}
f(\rho \cos \varphi, \rho \sin \varphi)=\rho^{n} \sum_{k=0}^{n} a_{k} \cos ^{k} \varphi \sin ^{n-k} \varphi \\
=\rho^{n} \sum_{k=0}^{n} a_{k}\left(\frac{e^{-i \varphi}+e^{i \varphi}}{2}\right)^{k}\left(\frac{e^{i \varphi}-e^{-i \varphi}}{2 i}\right)^{n-k} \\
=\frac{\rho^{n}}{2^{n}}\left\{\left[e^{i n \varphi} \sum_{k=0}^{n} a_{k} i^{k-n}+e^{-i n \varphi} \sum_{k=0}^{n} a_{k} i^{k-n}(-1)^{n-k}\right]\right. \\
\left.\quad+\left[e^{(n-2) \varphi} \sum_{k=0}^{n} a_{k} i^{k-n}(2 k-n)+e^{-i(n-2) \varphi} \sum_{k=0}^{n} a_{k} i^{k-n}(2 k-n)(-1)^{n-k}\right]+\cdots\right\} \\
=2^{-n} \rho^{n}\left\{A_{n}(f, \varphi)+A_{n-2}(f, \varphi)+\cdots\right\} .
\end{aligned}
$$

Положим

$$
I_{0}(f)=\sum_{k=0}^{n} a_{k} i^{k}, \quad I_{1}(f)=\sum_{k=0}^{n} a_{k} i^{k}(2 k-n) .
$$


ТЕорема 1. Для любого $f(x, y)$ из (1) $и$ любого многочлена $P(x, y)$ из $\mathscr{P}_{n-1}$ справедлива оченка

$$
\|f(x, y)-P(x, y)\|_{p} \geqslant 2^{1 / p+1-n}(p n+2)^{-1 / p}\|\cos \varphi\|_{p}\left|I_{0}(f)\right|, \quad p \geqslant 1 .
$$

ДоказАТЕЛьство. Так как

$$
\|f-P\|_{p}^{p}=\frac{1}{\pi} \iint_{B}|f(x, y)-P(x, y)|^{p} d x d y
$$

то переходя к полярньм координатам, найдем

$$
\|f-P\|_{p}^{p}=2 \int_{0}^{1} \rho d \rho \frac{1}{2 \pi} \int_{0}^{2 \pi}|f(\rho \cos \varphi, \rho \sin \varphi)-P(\rho \cos \varphi, \rho \sin \varphi)|^{p} d \varphi .
$$

При любом $\rho, 0 \leqslant \rho \leqslant 1$, относительно $\varphi$ тригонометрический полином $P(\rho \cos \varphi, \rho \sin \varphi)$ имеет порядок, не превосходящий $n-1$. Оценим внутренний интеграл по неравенству (4). Из определения (5) получим

$$
\|f-P\|_{p}^{p} \geqslant 2 \int_{0}^{1} \rho d \rho \cdot \rho^{p n} \cdot 2^{-p n}\left\|A_{n}(f, \varphi)\right\|_{p}^{p}=2^{1-p n}(p n+2)^{-1}\left\|A_{n}(f, \varphi)\right\|_{p}^{p} .
$$

Поскольку числа

$$
\sum_{k=0}^{n} a_{k} i^{k-n}, \quad \sum_{k=0}^{n} a_{k} i^{k-n}(-1)^{n-k}
$$

комплексно-сопряжены, то

$$
\left\|A_{n}(f, \varphi)\right\|_{p}^{p}=\left\|e^{i n \varphi} I_{0}(f)+e^{-i n \varphi} \overline{I_{0}(f)}\right\|_{p}^{p}=2^{p}\left|I_{0}(f)\right|^{p}\|\cos \varphi\|_{p}^{p}
$$

Подставляя полученное соотношение в (7), приходим к оценке (6). Теорема доказана.

СЛЕДСТВИЕ 1. При любом $k \in \mathbb{N}$

$$
E_{n-1}\left(x^{k} y^{n-k}\right) \geqslant 2^{1-n}
$$

При $p=\infty$ оценка (6) принимает вид

$$
\|f(x, y)-P(x, y)\| \geqslant 2^{1-n}\left|I_{0}(f)\right|
$$

Если в последовательности $\left\{a_{k}\right\}_{0}^{n}$ одно из чисел положить равным 1 , а остальные равными 0 , т.е. $f(x, y)=x^{k} y^{n-k}$, то очевидно $\left|I_{0}(f)\right|=1$. Таким образом, дан новьй вывод нижней оценки (3). 
СЛЕДСТВИЕ 2. Для любого однородного гармонического многочлена $f(x, y)$ порядка п июбого $p \geqslant 1$

$$
E_{n-1}(f)_{p}=\inf _{P \in \mathscr{P}_{n-1}}\|f(x, y)-P(x, y)\|_{p}=\|f(x, y)\|_{p}
$$

Действительно,

$$
f(x, y)=\rho^{n}(a \cos n \varphi+b \sin n \varphi) \equiv \rho^{n} A_{n}(f, \varphi),
$$

поэтому в силу (6)

$$
E_{n-1}(f)_{p} \geqslant\|f(x, y)\|_{p}=\sqrt{a^{2}+b^{2}} 2^{1 / p+1-n}(n p+2)^{-1 / p}\|\cos \varphi\|_{p} .
$$

Полагая $P(x, y)=0$, получаем ту же самую оценку сверху для $E_{n-1}(f)_{p}$. Отметим, что при $p=\infty$ данньй факт доказан в [2] в более сильной форме.

ЗАмЕчАнИЕ 1 . Пусть $g(x, y)$ - однородньй гармонический многочлен порядка $n-2$. Для $f(x, y)=\left(x^{2}+y^{2}\right) g(x, y)$ оценка (6) несодержательна: $E_{n-1}(f)_{p} \geqslant 0$. Эта ситуация наблюдается и в более общих случаях. Небольшое изменение рассуждений, используемых при доказательстве теоремы 1 , позволяет получать новые нижние оценки. Например, при $n>4$

$$
E_{n-1}^{p}\left(\left(x^{2}+y^{2}\right) g(x, y)\right)_{p} \geqslant 2^{(1-n) p}\left|I_{1}(f)\right|^{p}\|\cos \varphi\|_{p}^{p} \inf _{Q_{n-2}} \int_{0}^{1} \rho\left|\rho^{n}-Q_{n-2}(\rho)\right|^{p} d \rho
$$

где нижняя грань берется по алгебраическим (относительно $\rho$ ) многочленам порядка $n-2$. Вычисление последних оказьвается затруднительным.

ЗАмЕчАниЕ 2. Как показано вьше, приближение любого однородного гармонического многочлена на двумерном шаре тривиально. Если рассмотреть $d$-мерньй шар из $\mathbb{R}^{d}$

$$
B^{d}=\left\{x=\left(x_{1}, \ldots, x_{d}\right) \in \mathbb{R}^{d}: x_{1}^{2}+\cdots+x_{d}^{2} \leqslant 1\right\},
$$

то это утверждение не имеет места при $d \geqslant 3$ уже для многочленов второго порядка, хотя для некоторых, например, $f\left(x_{1}, \ldots, x_{d}\right)=x_{1} \cdots x_{d}$, справедливо [6]. Пусть

$$
f(x)=(d-1) x_{1}^{2}-x_{2}^{2}-\cdots-x_{d}^{2},
$$

очевидно,

и

$$
\Delta f=\frac{\partial^{2} f}{\partial x_{1}^{2}}+\cdots+\frac{\partial^{2} f}{\partial x_{d}^{2}} \equiv 0
$$

$$
\max _{x \in B^{d}} f(x)=d-1, \quad \min _{x \in B^{d}} f(x)=-1 .
$$

Полагая $P(x)=d / 2-1$, найдем, что при $x \in B^{d}$

$$
f(x)-P(x)=(d-1) x_{1}^{2}-x_{2}^{2}-\cdots-x_{d}^{2}-\frac{d}{2}+1 \leqslant(d-1) x_{1}^{2}-\frac{d}{2}+1 \leqslant \frac{d}{2}
$$

и отсюда

$$
f(x)-P(x)=d x_{1}^{2}-\left(x_{1}^{2}+\cdots+x_{d}^{2}\right)-\frac{d}{2}+1 \geqslant-\frac{d}{2} .
$$

Следовательно, при $d \geqslant 3$

$$
\max _{x \in B^{d}}|f(x)-P(x)| \leqslant \frac{d}{2}<\max _{x \in B^{d}}|f(x)|=d-1 .
$$

В дальнейшем будем рассматривать лишь случай $p=\infty$. Дадим новый способ оценок уклонения (2) сверху. Он позволяет находить точные значения $E_{n-1}(f)$ в случае, когда норма $A_{n}(f, \varphi)$ равна “достаточно большой доле" нормы граничного значения $\left.f\right|_{\Gamma}$. 
TeOpema 2. Пyсmb $n \geqslant 3 u$

$$
\|f(\cos \varphi, \sin \varphi)\| \leqslant\left(\frac{n}{2}-1\right)\left\|A_{n}(f, \varphi)\right\| .
$$

Тогда

$$
E_{n-1}(f)=2^{1-n}\left|I_{0}(f)\right| \text {. }
$$

ДокАЗАТЕльСтво. Нижняя оценка для (9) получена в (8); найдем верхнюю. Следуя (5), положим

$$
P(x, y)=2^{-n} \rho^{n-2}\left\{A_{n-2}(f, \varphi)+A_{n-4}(f, \varphi)+\cdots\right\} .
$$

Поскольку выражения

$$
\rho^{n-2} \cos (n-2) \varphi, \quad \rho^{n-2} \sin (n-2) \varphi, \quad \rho^{n-2} \cos (n-4) \varphi, \quad \rho^{n-2} \sin (n-4) \varphi, \quad \ldots
$$

являются алгебраическими многочленами из $\mathscr{P}_{n-2}$, то $P(x, y) \in \mathscr{P}_{n-2}$. Для произвольного $\rho, 0 \leqslant \rho \leqslant 1$, оценим уклонение

$$
I(\rho, \varphi)=f(\rho \cos \varphi, \rho \sin \varphi)-P(\rho \cos \varphi, \rho \sin \varphi) .
$$

Из представления (5) получим

$$
I(\rho, \varphi)=2^{-n}\left[\rho^{n} A_{n}(f, \varphi)+\left(\rho^{n}-\rho^{n-2}\right)\left(A_{n-2}(f, \varphi)+A_{n-4}(f, \varphi)+\cdots\right)\right],
$$

откуда

$$
\begin{aligned}
\max _{\varphi}|I(f, \varphi)| & \leqslant 2^{-n}\left[\rho^{n}\left\|A_{n}(f, \varphi)\right\|+\left(\rho^{n-2}-\rho^{n}\right)\left\|f(\cos \varphi, \sin \varphi)-A_{n}(f, \varphi)\right\|\right] \\
& \leqslant 2^{-n}\left[\rho^{n}\left\|A_{n}(f, \varphi)\right\|+\left(\rho^{n-2}-\rho^{n}\right)\left(\|f(\cos \varphi, \sin \varphi)\|+\left\|A_{n}(f, \varphi)\right\|\right)\right] .
\end{aligned}
$$

По условию теоремы

$$
\begin{aligned}
\max _{\varphi} I(f, \varphi) & \leqslant 2^{-n}\left\{\rho^{n}\left\|A_{n}(f, \varphi)\right\|+\left(\rho^{n-2}-\rho^{n}\right) \frac{n}{2}\left\|A_{n}(f, \varphi)\right\|\right\} \\
& =2^{1-n}\left|I_{0}(f)\right| \cdot\left\{\frac{n}{2} \rho^{n-2}+\left(1-\frac{n}{2}\right) \rho^{n}\right\} .
\end{aligned}
$$

Нетрудно проверить, что двучлен

$$
\frac{n}{2} \rho^{n-2}+\left(1-\frac{n}{2}\right) \rho^{n}
$$

возрастает на интервале $(0,1)$. Поэтому наибольшее значение, равное 1 , он достигает при $\rho=1$. Значит,

$$
E_{n-1}(f) \leqslant \max _{\varphi}|f(\cos \varphi, \sin \varphi)-P(\cos \varphi, \sin \varphi)| \leqslant 2^{1-n}\left|I_{0}(f)\right| .
$$

Теорема доказана.

Для $f(x, y)=x^{n} y^{m}$ единственность многочлена наилучшего приближения из $\mathscr{P}_{n+m-1}$ имеется [2] лишь в двух случаях:

a) $n=0$ или $m=0$;

b) $n=m=1$. 
В [2], [7] даны некоторые способы построения многочленов наилучшего приближения. Непосредственной проверкой легко убедиться, что семейство многочленов из $\mathscr{P}_{4}$

$$
P(x, y)=\frac{3}{16}\left[(1+\theta) x y\left(x^{2}+y^{2}\right)+\theta x y\right], \quad 0 \leqslant \theta \leqslant \frac{5}{3}
$$

наилучшим образом приближает на $B f(x, y)=x^{3} y^{3}$,

$$
\|f(x, y)-P(x, y)\| \leqslant 2^{-5} .
$$

На этом примере покажем разницу в выборе наилучших многочленов из [2] и из теоремы 2. В [2] выбиралось $\theta=5 / 3$, а в настоящей работе $\theta=0$.

В заключение автор присоединяется к точке зрения В. Герхарта и М. Реймера: вычисление точных значений $E_{n-1}(f)$ для произвольного $f(x, y)$ из $(1)$ затруднительно. Прогресс видится в новых способах построения многочленов наилучшего приближения. Здесь к успеху приводят даже интерполяционные конструкции (см. [8], [9] с подробностями). Однако за исключением некоторых простейших случаев в пространстве $L_{1}(B)$ (аналог теоремы Коркина-Золотарева [10]) построение многочленов наименьшего уклонения неизвестно.

\section{СПИСОК ЦИТИРОВАННОЙ ЛИТЕРАТУРЫ}

[1] Чебышев П. Л. Полн. собр. сочинений. М.-Л.: Изд-во АН СССР, 1948.

[2] Gearhart W. B. Some Chebyshev approximations by polynomials in two variables // J. Approx. Theory. 1973. V. 8. P. 195-209.

[3] Rivlin T. J., Shapiro H.S. A unified approach to certain problems of approximation and minimization // J. Soc. Indust. Appl. Math. 1961. V. 9. P. 670-699.

[4] Reimer M. Constructive Theory of Multivariate Functions. With an Application to Tomography. Mannheim: Bibliographisches Institut, 1990.

[5] Бернштейн С. Н. Полн. собр. сочинений. Т. 1. М.: Изд-во АН СССР, 1952.

[6] Андреев Н. Н., Юдин В. А. Наименее уклоняющиеся от нуля многочлены и кубатурные формулы Чебьшевского типа // Тр. МИАН. 2001. Т. 232. С. 45-57.

[7] Reimer M. On multivariate polynomials of least deviation from zero on the unit ball // Math. Z. 1977. V. 153. P. 51-58.

[8] Bos L. On Kergin interpolation in the disk // J. Approx. Theory. 1983. V. 37. P. 251-261.

[9] Majer U. On best approximation of the monomials on the unit ball of $\mathbb{R}^{r} / /$ J. Approx. Theory. 1998. V. 72. P. 74-81.

[10] Коркин А. Н., Золотарев Е. И. Sur un certain minimum // Полн. собр. соч. Е. И. Золотарева. Т. 1. Л.: Изд-во АН СССР, 1931. С. 138-153. 